\title{
Wind-PV-Thermal Power Aggregator in Electricity Market
}

\author{
I.L.R. Gomes ${ }^{1,2}$, R. Laia ${ }^{2}$, H.M.I. Pousinho ${ }^{2}$, R. Melicio ${ }^{1,2}$, V.M.F. Mendes ${ }^{3,4}$ \\ ${ }^{1}$ IDMEC, Instituto Superior Técnico, Universidade de Lisboa, Lisbon (ruimelicio@gmail.com) \\ ${ }^{2}$ ICT, Universidade de Évora, Escola de Ciências e Tecnologia, Departamento de Física \\ ${ }^{3}$ CISE, Electromechatronic Systems Research Centre, Universidade da Beira Interior, Portugal \\ ${ }^{4}$ Instituto Superior of Engenharia de Lisboa, Lisbon, Portugal
}

\begin{abstract}
This paper addresses the aggregation of wind, photovoltaic and thermal units with the aim to improve bidding in an electricity market. Market prices, wind and photovoltaic powers are assumed as data given by a set of scenarios. Thermal unit modeling includes start-up costs, variables costs and bounds due to constraints of technical operation, such as: ramp up/down limits and minimum up/down time limits. The modeling is carried out in order to develop a mathematical programming problem based in a stochastic programming approach formulated as a mixed integer linear programming problem. A case study comparison between disaggregated and aggregated bids for the electricity market of the Iberian Peninsula is presented to reveal the advantage of the aggregation.
\end{abstract}

Keywords: Aggregator; day-ahead market; mixed integer linear programming; stochastic programming; wind-pv-thermal units; variable renewables.

\section{Introduction}

The usage of non-renewable energy sources has been assumed as responsible for global warming and climate change, hovering a view of a worldwide sustainable development where renewable energy sources are the way of the future to be favored in the electricity industry [1]. But the variability of the available input energy in wind or photovoltaic (PV) renewable power units are doomed to fluctuate hastily, implying for the exploitation of the electricity industry uncertainty in energy delivering. If the rated power of these units is small in comparison with the power of non-fluctuating units in a power system, the impact of the fluctuation can be neglected. Else, the impact is of paramount significance and actions are to be taken. So, with the increasing penetration of wind or PV renewable power units, menaces to system reliability and security are to be expected $[2,3]$. Additional ancillary service, i.e., more schedule of power of reserve units ready to be called if needed, must be incurred to recover an acceptable level of operation reliability and minimize the associated impact charge [4]. As the electricity industry follows in the way of Internet of Things (IoT) and of Energy 4.0, aggregation platforms are essential for distributed energy resources, storage, and multiple costumer loads like microgrids [5]. An aggregation platform for wind, photovoltaic and thermal units can be viewed as tool for an aggregator to act as facilitator for offering energy in an electricity market [6]. Strategic aggregation of power units is on an increasing interest for researchers and on demand from the electricity industry. The electricity industry not only requires adequate management information systems to improve profit in electricity market environment, but 
also to be in an electricity market environment is required to have enough capacity and ability to satisfy assumed compromised of delivering energy in due time [7].

Aggregation is able to harvest better bidding, accommodating fluctuation of variable renewables, carrying favorable imbalance while increasing the reliability and improving market performance. A convenient management of uncertainties due to the power of wind and PV units may reduce the deviations of energy and reduce the level of power required by thermal units in a wind-PV-thermal commitment. This convenient management for the wind-PV-thermal commitment is the issue in research in this paper in order to develop a computer procedure. The contribution is concerned with the: definition and advantages of a Wind-PV-Thermal power aggregator and the modeling in what concerns the strategy of bidding in electricity market. Market prices, wind and photovoltaic powers are data for the proposed computer procedure given by a set of scenarios. The thermal units modeling has continuous and integer decision variables in order to include a modeling for the startup costs, variables costs and bounds due to technical operation constraints, such as: ramp up/down limits and minimum up/down time limits. The modeling is carried out in order to develop a management aggregation procedure based on a stochastic programming approach formulated as a mixed integer linear mathematical programming problem. A case study comparison between disaggregated and aggregated biddings shows the benefit of the aggregation proposed in this paper, particularly, the improvement in the profit.

\section{Relationship to Resilient Systems}

The electricity market approaches tend to maximize the economic efficiency, but may lead to deterioration on the resilience of energy systems [8]. United Nations has highlighted the importance of considering resilience in the designing of systems and infrastructures with the goal of being able to tolerate external shocks that might occur in the future [9]. Resilience of system is a capacity of being able of absorbing shocks and reorganizing while undergoing change to retain essentially the same function. Distinguished from sustainability, resilience is related to how the system responds to disturbance or non-linear dynamics [10]. A resilient energy system has the capacity to quickly recover from external shocks and to provide alternative means of maintaining a satisfactory level of services to consumers in the event of external disturbances [11]. In [12] is discussed the importance of ensuring the resilience of energy supply because the infrastructures have susceptibility to large-scale failures caused by unforeseen scenarios. Namely, natural disaster and technical failures. The future smart grid ambient and cyber physical systems have a layered architecture of a cyber infrastructure accessing resilient power applications able to give security and reliability, having the ability to act in order to maintain and correct infrastructure components without affecting the service [13]. Aggregated platforms have to be embodied with tools to allow resilience and contribute to improve the economic and technical performance in decision-making. This paper is a research on one of these tools customized for the Wind-PV-Thermal power aggregator, adding economic and technical value to the platform, giving the ability to manage the set of resources in coordination, harmonizing different functionality to assemble condition to be in an electricity market, achieving effectiveness, efficiency and efficacy. Considering the scenario of increasing penetration of variable-renewables the electrical system of Iberian Peninsula have to lead with some problems of resilience, since the fluctuations of variable-renewables may not be accommodated. To lead with this issue 
higher levels of interconnection with another regions should be verified according to European Union reports.

\section{State of the Art}

The former unit commitment in a view of the regulated electricity market is set with a paradigm formally given by an optimization problem related to the minimization of the operating costs while meeting the load demand and subjected to other constraints. This type of unit commitment is known as cost-based unit commitment. The deregulated electricity market paradigm brought new opportunities for power producers in an environment where the optimization is related to the maximization of profit. This new paradigm is known as price-based unit commitment [14]. The unit commitment problem can be divided into two sub-problems: i) the unit on/off scheduling sub-problem period; and ii) the economic dispatch sub-problem, which determines the power output of units in each period of the time horizon [15]. A line of research on variable-renewables and thermal unit commitment is raising up with the increasing penetration of wind or PV renewable power units. Some research is concerned with time resolution, i.e., the impact of considering sub-hourly periods in the commitment $[16,17]$. In order to cope the uncertainties of variable-renewables is proposed the use with storage technologies [18] or financial options [19]. Another relevant research is concerned with the application of stochastic optimization methods and is expected to achieve a significant application in what concerns optimum bidding strategy for aggregation [20,21]. Several of popular algorithms using stochastic optimization in unit commitment problems are based on decomposition techniques.

\section{Wind-PV-Integrated Unit Commitment Problem}

The Wind-PV-Thermal power aggregator owns wind turbines, PV modules and thermal units. The first two types of units have unpredictable and stochastic nature and are categorized as variable-renewable sources of energy, imposing challenges to the unit commitment problem. These challenges are linked with the determination of the optimal day-ahead bid under wind and PV power uncertainties and the decision for the optimal generation of thermal units. This paper addresses a two-stage stochastic programming approach that takes into account the uncertainties related to the variable renewables and the market prices by scenarios. The scenarios can be expressed by means of a scenario tree, but in the scope of the paper the scenarios are considered as given data attained by the use of an adequate tool. The stochastic programming is reformulated in an admissible way as a mixed-integer linear programming (MILP) approach to benefit from the available and well proved practical commercial optimization solvers. In a two-stage stochastic optimization decision variables are categorized into first and second stage decisions: a) The first stage decisions, which are known as here and know decisions, are made before the realization of uncertainties which in this paper are: wind power, PV power and market prices, including imbalance prices. The thermal unit commitment and the hourly bids are the first-stage decisions. The objective is the maximization of the profit of the production aggregator in the first stage; b) The second stage decisions, which are known as wait and see decisions are made after the realization of the aforementioned uncertainty decisions. Second stage decisions are related to the economic dispatch, i.e., the power output of units in each period of the planning horizon. The objective of the optimization problem is to maximize the realized expected profit of the production aggregator in the second stage. Normally is assumed that the operating costs of variable 
renewables are negligible in comparison with the one of thermal units. Therefore, the total operating cost to be considered for the aggregation is the cost due to the operation of the thermal unit. The total operation cost $O P_{\text {sit }}$ is stated as follows:

$$
O P_{s i t}=G C_{s i t}+S U C_{s i t}+S D C_{t} z_{i t} \quad \forall s, \quad \forall i, \quad \forall t
$$

In (1) the three terms are measures for the generation cost $G C_{\text {sit }}$, start-up cost $S U C_{\text {sit }}$, and shut-down cost $S D C_{\text {sit }}$. The generation cost $G C_{\text {sit }}$ is stated as follows:

$$
G C_{s i t}=A_{i} u_{i t}+d_{s i t} \quad \forall s, \quad \forall i, \quad \forall t
$$

In (2) the two terms are the fixed cost and the variable cost of unit $i$. The generation cost is normally approximated by a quadratic function, but this paper follows the model for an approximation as a piecewise linear function stated as follows:

$$
\begin{gathered}
d_{s i t}=\sum_{l=1}^{L} F_{i}^{l} \delta_{s i t}^{l} \quad \forall s, \quad \forall i, \quad \forall t \\
p_{s i t}=p_{i}^{\min } u_{i t}+\sum_{l=1}^{L} \delta_{s i t}^{l} \quad \forall s, \quad \forall i, \quad \forall t \\
\left(T_{i}^{1}-p_{i}^{\min }\right) t_{s i t}^{1} \leq \delta_{s i t}^{1} \quad \forall s, \quad \forall i, \quad \forall t \\
\delta_{s i t}^{1} \leq\left(T_{i}^{1}-p_{i}^{\min }\right) u_{i t} \quad \forall s, \quad \forall i, \quad \forall t \\
\left(T_{i}^{l}-T_{i}^{l-1}\right) t_{\text {sit }}^{l} \leq \delta_{\text {sit }}^{l} \quad \forall s, \quad \forall i, \quad \forall t, \quad \forall l=2, \ldots, L-1 \\
\delta_{s i t}^{l} \leq\left(T_{i}^{l}-T_{i}^{l-1}\right) t_{s i t}^{l-1} \quad \forall s, \quad \forall i, \quad \forall t, \quad \forall l=2, \ldots, L-1 \\
0 \leq \delta_{s i t}^{L} \leq\left(p_{i}^{\max }-T_{s i t}^{L-1}\right) t_{s i t}^{L-1}
\end{gathered}
$$

In (3) the variable cost function is given by the sum of the product of the slope of each segment, $F_{i}^{l}$, by the segment power $\delta_{s i t}^{l}$. In (4) the power generation of the unit is given by the minimum power generation plus the sum of the segment powers associated with each segment. The binary variable $u_{i t}$ ensures that the power generation is equal to 0 if the unit is in the state offline. In (5) to (9) are defined the limits of power generation in each segment. The start-up cost $S U C_{\text {sit }}$ are normally represented by means of an exponential function. This exponential function is approximated by a stair wise function with a convenient selection of the number of intervals and is stated as follows:

$$
S U C_{s i t} \geq K_{i}^{\beta}\left(u_{i t}-\sum_{r=1}^{\beta} u_{i t-r}\right) \quad \forall s, \quad \forall i, \quad \forall t
$$

In (10) the right term is the imputed cost for a unit to have a start-up, i.e., if the unit is in the state online at hour $t$, and in the previous $\beta$ hour has been in the state offline, the expression in parentheses is equal to 1 , a start-up happens and the respective cost is has to be considered. The constraints to limit the power of unit $i$ are stated as follows:

$$
\begin{gathered}
p_{i}^{\min } u_{i t} \leq p_{s i t} \leq p_{s i t}^{\max } \quad \forall s, \quad \forall i, \quad \forall t \\
p_{s i t}^{\max } \leq p_{i}^{\max }\left(u_{i t}-z_{i t+1}\right)+S D z_{i t+1} \quad \forall s, \quad \forall i, \quad \forall t \\
p_{s i t}^{\max } \leq p_{s i t-1}^{\max }+R U u_{i t-1}+S U y_{i t} \quad \forall s, \quad \forall i, \quad \forall t
\end{gathered}
$$




$$
p_{s i t-1}-p_{s i t} \leq R D u_{i t}+S D z_{i t} \quad \forall s, \quad \forall i, \quad \forall t
$$

In (11) for unit $i$ at hour $t$ the variable $p_{\text {sit }}$ represents the energy bid limited by the maximum power $p_{\text {sit }}^{\max }$ of unit $i$ at hour $t$. The maximum power $p_{\text {sit }}^{\max }$ value takes into consideration the actual capacity unit $i$ at hour $t$, considering start-up, shut-down ramp rate limits and ramp-up limit. In (12) to (14) the relation between the start-up and shutdown variables of unit $i$ is imposed, using binary variables. The modeling for the minimum up and down time constraints is based on constraints imposed on the binary variables. The minimum up time constraints are stated as follows:

$$
\begin{gathered}
\sum_{i=1}^{N_{i}}\left(1-u_{i t}\right)=0, \quad \forall i, \quad \forall t \\
\sum_{i=k}^{k+U T_{i}-1} u_{i t} \geq U T_{i} y_{i t} \quad, \forall i, \quad \forall k=N_{i}+1 \ldots T-U T_{i}+1 \\
\sum_{t=k}^{T}\left(u_{i t}-z_{i t}\right) \geq 0 \quad, \quad \forall i, \quad \forall k=T-U T_{i}+2 \ldots T \\
N_{i}=\min \left\{T,\left(U T_{i}-U_{i 0}\right) u_{i 0}\right\}
\end{gathered}
$$

In (15) to (17) once the unit is on remain on until reaching minimum up time. A unit start-up up is kept on by $U T_{i}$ hours by (16). Similarly, the minimum down time constraints are stated as follows:

$$
\begin{gathered}
\sum_{t=1}^{J_{i}} u_{i t}=0, \quad \forall i, \quad \forall t \\
\sum_{t=k}^{k+D T_{i}-1}\left(1-u_{i t}\right) \geq D T_{i} z_{i t}, \quad \forall i, \quad \forall k=J_{i}+1 \ldots T-D T_{i}+1 \\
\sum_{t=k}^{T}\left(1-u_{i t}-z_{i t}\right) \geq 0, \quad \forall i, \quad \forall k=T-D T_{i}+2 \ldots T \\
J_{i}=\min \left\{T,\left(D T_{i}-s_{s i 0}\right)\left(1-u_{i 0}\right)\right\}
\end{gathered}
$$

In (18) to (20) the unit once off stays minimum down time ours off. The start-up and shutdown coupling constraints between the binary variables and the total power of the thermal units are respectively stated as follows:

$$
\begin{gathered}
y_{i t}-z_{i t}=u_{i t}-u_{i t-1}, \quad \forall i, \quad \forall t \text { and } y_{i t}+z_{i t} \leq 1, \quad \forall i, \quad \forall t \\
p_{s t}^{g}=\sum_{i=1}^{I} p_{s i t} \quad \forall s, \quad \forall t
\end{gathered}
$$

In (21) is imposed a restriction on start-up and shutdown variables so that a unit is not allowed to start up or shut down simultaneously.

Imbalance prices, i.e., up and down-regulation prices, are given in the Iberian balancing market through a double price procedure, computing how the prices for deviations are accommodated in a view of both the system imbalance and producer imbalance as shown in Table 1. In Table $1 \lambda_{t}^{+}$and $\lambda_{t}^{-}$are positive and negative imbalance prices, respectively, $\lambda_{t}^{D}$ is the day-ahead market price, $\lambda_{t}^{U P}$ and $\lambda_{t}^{D N}$ are the up and the down- 
regulation prices, respectively. $\lambda_{t}^{+}$and $\lambda_{t}^{-}$can be expressed by means of price ratios $p r_{t}^{+}$and $p r_{t}^{-}$.

Table 1. Imbalance prices

\begin{tabular}{cccc}
\hline & & \multicolumn{2}{c}{ System imbalance } \\
\cline { 2 - 4 } & & Negative & Positive \\
\hline \multirow{2}{*}{$\begin{array}{c}\text { Power producer } \\
\text { imbalance }\end{array}$} & Negative & $\lambda_{t}^{-}=\max \left(\lambda_{t}^{D}, \lambda_{t}^{U P}\right)$ & $\lambda_{t}^{-}=\lambda_{t}^{D}$ \\
\cline { 2 - 4 } & Positive & $\lambda_{t}^{+}=\lambda_{t}^{D}$ & $\lambda_{t}^{+}=\min \left(\lambda_{t}^{D}, \lambda_{t}^{D N}\right)$ \\
\hline
\end{tabular}

The main objective of the production aggregator wind-PV-integrated unit commitment is to maximize the total expected profit in the day-ahead market. The objective function is stated as follows:

$$
\sum_{s=1}^{N_{S}} \sum_{t=1}^{N_{T}} \frac{1}{N_{S}}\left[\left(\lambda_{s t}^{D} P_{s t}^{\text {Aggregated }}+\lambda_{s t}^{D} p r_{s t}^{+} d_{s t}^{+}-\lambda_{s t}^{D} p r_{s t}^{-} d_{s t}^{-}\right)-\sum_{i=1}^{I} O P_{s i t}\right] \quad \forall s, \quad \forall t
$$

Subject to:

$$
\begin{gathered}
0 \leq P_{s t}^{\text {Aggregated }} \leq \sum_{i=1}^{I} p_{s i t}^{\max }+p^{W \max }+p^{P V \max } \quad \forall s, \quad \forall t \\
d_{s t}=\left(p_{s t}^{g}+p_{s t}^{W}+p_{s t}^{P V}-P_{s t}^{\text {Aggregated }}\right) \quad \forall s, \quad \forall t \\
d_{s t}=d_{s t}^{+}-d_{s t}^{-} \quad \forall s, \quad \forall t \text { and } 0 \leq d_{s t}^{+} \leq p_{s t}^{g}+p_{s t}^{W}+p_{s t}^{P V} \quad \forall s, \quad \forall t
\end{gathered}
$$

In (23) the four terms of the objective function are the per scenario profit of the dayahead bid, the outcome incurred by the respective imbalances, and total thermal operating cost, respectively. Offer curves are normally subjected to a non-decreasing dependence stated as follows:

$$
\left(P_{s t}^{\text {Aggregated }}-P_{s^{\prime} t}^{\text {Aggregated }}\right)\left(\lambda_{s t}^{D}-\lambda_{s^{\prime} t}^{D}\right) \geq 0 \quad \forall s, s^{\prime}, \quad \forall t
$$

In (27) as the price is incremented the power increment is imposed as non-negative.

\section{Case Study}

The case study is for a Wind-PV-Thermal power aggregator owning a wind system with a power rated of $100 \mathrm{MW}$, a PV system with $100 \mathrm{MW}$ and 2 thermal units with $285 \mathrm{MW}$. The day-ahead market prices and the price ratios $\mathrm{pr}_{t}^{+}$e $p r_{t}^{-}$are the ones reported in [22]. The bidding is for a $24 \mathrm{~h}$ horizon on an hourly basis. The scenarios considered are 5 for wind power, 5 for PV power, 5 for day-ahead market prices and 5 for price ratios. The data for the thermal units used in this research work are reported in [21]. The day-ahead market price scenarios, the wind powers scenarios and PV power scenarios are shown in Fig. 1.

The unit commitment of thermal units and the expected profit of disaggregated and aggregated coordination are shown respectively in Table 2 and in Table 3.

In Table 2 unit 1 is on online state (1) in all the 24 hours, while unit 2 is offline (0) in hour 1 and 2, then has a start-up and stays online. Table 3 shows the expected profit 
for disaggregated and aggregated coordination. The aggregation allows obtain an increase in performance of about $2.5 \%$ in profit.
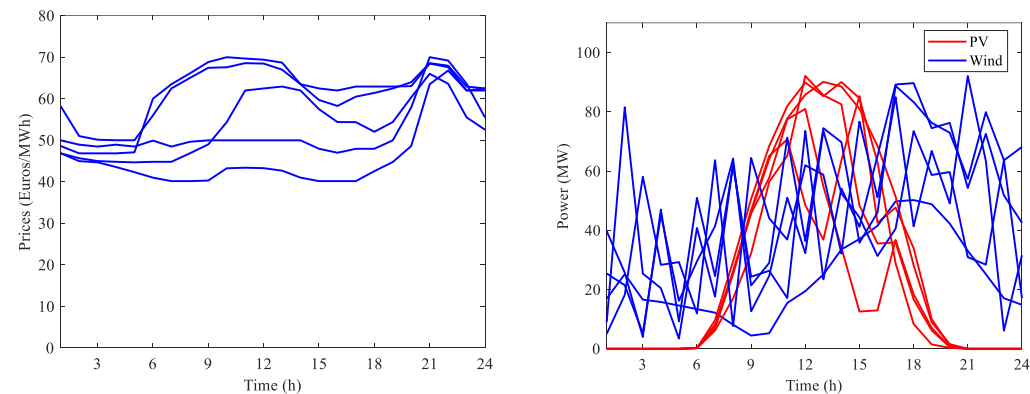

Fig 1. Scenarios: Left, day-ahead market price; Right, PV (red lines), Wind (blue lines).

Table 2. Unit status

\begin{tabular}{cccccccccccccc}
\hline Hour & 1 & 2 & 3 & 4 & 5 & 6 & 7 & 8 & 9 & 10 & 11 & 12 \\
\hline Unit 1 status & 1 & 1 & 1 & 1 & 1 & 1 & 1 & 1 & 1 & 1 & 1 & 1 \\
\hline Unit 2 status & & 0 & 0 & 1 & 1 & 1 & 1 & 1 & 1 & 1 & 1 & 1 & 1 \\
\hline Hour & 13 & 14 & 15 & 16 & 17 & 18 & 19 & 20 & 21 & 22 & 23 & 24 \\
\hline Unit 1 status & 1 & 1 & 1 & 1 & 1 & 1 & 1 & 1 & 1 & 1 & 1 & 1 \\
\hline Unit 2 status & 1 & 1 & 1 & 1 & 1 & 1 & 1 & 1 & 1 & 1 & 1 & 1
\end{tabular}

Table 3. Expected profit

\begin{tabular}{cr}
\hline Case & Expected profit $[€]$ \\
\hline Wind disaggregated & 53411.9 \\
Photovoltaic disaggregated & 33515.0 \\
Thermal disaggregated & 130217.2 \\
Wind Photovoltaic Thermal - disaggregated & 217144.2 \\
Wind Photovoltaic Thermal - aggregated & 222671.5 \\
\hline
\end{tabular}

\section{Conclusion}

Aggregation platforms deliver an increase of resilience on the aggregated systems since better decisions are made in the bidding process while achieving an adequate technical performance. The wind and photovoltaic uncertainties and market uncertainties, including day-ahead market prices and imbalance prices are considered as input data given by scenarios for the proposed platform. A mixed-integer stochastic optimization approach is proposed to address the problem of aggregation in an optimum way. The result of the proposed approach is the optimum commitment of the units and bidding. The aggregation can improve the aggregator's profit in $2.5 \%$ as shown by the case study. The aggregation of wind systems, PV systems and thermal units using stochastic optimization allows to manage uncertainties in a more convenient way. Although this management is not able to ensure null imbalances, the management harmonizes the uncertainties to deliver imbalances in accordance with the aim of accessing optimum profit in the electricity market. This management with aggregation has proved to be better than the previous single stochastic optimization works. The consideration of energy storage devices in a future work can improve the reliability and the profit of the aggregated system proposed in this research work, in the way that energy can be stored in periods of low market prices and sold at periods of likely high 
prices. In addition stored energy can be used to compensate the fluctuations of the variable-renewables.

\section{Acknowledgments}

To thank the Millennium BCP Foundation for the financial support; and Foundation for Science and Technology-FCT project LAETA ref: UID/EMS/50022/2013.

\section{References}

1. Destek, M.A., Aslan, A.: Renewable and non-renewable energy consumption and economic growth in emerging economies: Evidence from bootstrap panel causality. Ren. Energy 111,757-763 (2017)

2. González-Aparicio, I., Zucker A.: Impact of wind power uncertainty forecasting on the market integration of wind energy in Spain. Applied Energy 159, 334-49 (2015)

3. Purvins, A., Zubaryeva, A., Llorente, M., Tzimas, E., Mercier, A.: Challenges and options for a large wind power uptake by European electricicty system. Applied Energy 88(5), 1461-1469 (2011)

4. Ummels, B.C., Gibescu, M., Pelggrum, E., Kling, W.L., Brand, A.J.: Impacts of wind power on thermal generation unit commitment and dispatch. IEEE Transactions on Energy Conversion 22(1), 44-51 (2007)

5. Asmus, P.: Microgrids, virtual power plants and our distributed energy future. The Electricity Journal 23(10), 72-82 (2010)

6. Carreiro, A.M., Jorge, H.M., Antunes, C.H.: Energy management systems aggregators: a literature survey. Renewable and Sustainable Energy Reviews 73, 1160-1172 (2017)

7. Wang, Q., Zhang, C., Ding, Y., Xydis, G., Wang, J., Ostergaard, J.: Review of real-time electricity markets for integrating distributed energy resources and demand response. Applied Energy 138, 695-706 (2015)

8. Lietaer, B., Ulanowicz, R.E., Goemer, S.J., McLaren, N.: Is our monetray structure a systemic cause for a finacial instability? Evidence and remedies from nature. J. Futur. Stud 14 (3);89-108 (2010)

9. United Nations. Sustainable development goals. Available at: https://sustainabledevelopment.un.org/topics/sustainabledevelopmentgoals (2016)

10. Folke, C. Resilience: the emergence of a perspective for social-ecological systems analyses. Glob. Environ. Chang. 16 (3):253-267 (2006)

11. Kishita, Y., McLaren, B.C., Giurco, D., Aoki, K., Yoshizawa, G., Handoh, I.C.: Designing backcasting secnarios for resilient energy futures. Tech. Forecast. and Social Change 124, 114 125 (2017)

12. Lovins, A.B., Lovins, L.H. Brittle power: energy strategy for national security. Brick House Publishing Co., Inc., Andover, MA. 1982

13. Gomes, I.L.R, Pousinho, H.M.I, Melicio, R, Mendes, R.: Bidding and Optimization Strategies for Wind-PV Systems in Electrcicity Markets assisted by CPS. Energy Procedia 106, 111-121 (2016)

14. Shahidehpour, M., Yamin, H., Li, Z.: Market overview in electric power systems, in Market Operations in Electric Power Systems, New York: Wiley \& Sons Ltd. 2002

15. Wood, A.J., Wollenberg, B.F., Sheblé, G.B.: Power generation, operation, and control. 3rd ed. New York: Wiley. 2013

16. Deane, J.P., Drayton, G., Gallachóir, B.P.O.: The impact of sub-hourly modelling in power systems with significant levels of renewable generation. Applied Energy 113, 152-158 (2014)

17. Yang, Y., Wang, J., Guan, X., Zhai, Q.: Subhourly unit commitment with feasible energy delivery constraints. Applied Energy 96, 245-52 (2012)

18. Angarita, J.L., Usaola, J., Martinez-Crespo, J.: Combined hydro-wind generation bids in a poolbased electricity market. Electric Power Systems Research 79(7), 1038-1046 (2009).

19. Hedman, K.W., Sheble, G.B.: Comparing hedging methods for wind power: using pumped storage hydro units vs options purchasing. In: Proc. of International Conference on Probabilistic Methods Applied to Power Systems-PMAPS 2006, Sweden, 2006, 1-6.

20. Gomes, I.L.R., Pousinho, H.M.I, Melicio, R., Mendes, V.M.F.: Stochastic coordination of joint wind and photovoltaic systems with energy storage in day-ahead market. Energy 124, 310-320 (2017)

21. Laia, R., Pousinho, H.M.I., Melicio, R., Mendes, V.M.F.: Bidding strategy of wind-thermal energy producers. Renewable Energy 99, 673-681 (2016)

22. REE-Red Eléctrica de España. Available at: http://www.esios.ree.es/web-publica/ (2018) 